\title{
Mental health and dual sensory loss in older adults: a systematic review
}

\author{
Chyrisse Heine $^{1,2 *}$ and Colette J. Browning ${ }^{2}$ \\ ${ }^{1}$ Department of Human Communication Sciences, School of Allied Health, La Trobe University, Bundoora, VIC, Australia \\ 2 Primary Care Research Unit, School of Primary Health Care, Monash University, Notting Hill, VIC, Australia
}

\section{Edited by:}

Bamini Gopinath, University of

Sydney, Australia

\section{Reviewed by:}

Julie Maree Schneider, University of

Sydney, Australia

Walter Wittich, MAB-Mackay

Rehabilitation Centre, Canada

*Correspondence:

Chyrisse Heine, Department of Human Communication Sciences,

School of Allied Health, La Trobe

University, Plenty Rd., Bundoora,

VIC 3084, Australia

e-mail: chyrisse.heine@monash.edu
Mental health is a core component of quality of life in old age. Dual Sensory Loss (DSL; combined vision and hearing loss) is prevalent in older adults and has been correlated with decreased levels of well-being. This systematic review aimed to critically review and summarize the evidence from studies that examined the mental health of older adults with DSL. In accordance with the Preferred Reporting Items for Systematic Reviews (PRISMA) statement, specific databases were searched and eight articles were selected for final review. Seven studies investigated the association between DSL and depression or depressive symptoms, whilst one study explored the relationship between DSL and quality of life. No studies investigated the impact of DSL on anxiety. Overall, results of this review suggested that there is a significant relationship between DSL and decreased mental health with those with DSL either displaying depressive symptoms or being at risk for developing depression. Future research should focus on comparative studies of older people with and without sensory loss, as well as targeted studies of older people with dual sensory loss, that incorporate well-defined and valid measures of sensory loss and mental health.

Keywords: dual sensory loss, mental health, depression, systematic review

\section{BACKGROUND}

Mental health is a key component in the health and well-being of older adults and refers to the "state of well-being in which every individual realizes his or her own potential, can cope with the normal stresses of life, can work productively and fruitfully, and is able to make a contribution to her or his community" (World Health Organisation, 2013b, para. 1). Dual sensory loss (DSL) is the combined loss of vision and hearing and is a common contributor to the mental health and well-being of older adults (Heine and Browning, 2002).

DSL is particularly prevalent in the older adult population due to the gradual deterioration of their vision and/or hearing with advancing age (Davila et al., 2009), and particularly for veterans who are aged 85 years or older (Smith et al., 2008). In a Danish study of DSL rehabilitation clients Dammeyer (2013) found that DSL increased rapidly in those aged 65 years and over. In a similar Canadian study Wittich et al. (2012) found that $69 \%$ of DSL rehabilitation clients were aged 65 years and over. According to Caban et al. (2005) DSL increased from 1.3\% for 18 to 44 year olds to $6.6 \%$ in those aged 80 years and older. Schneider et al. (2012) estimated that $25 \%$ of participants aged 80 years and over in the Australian Blue Mountains Eye Study experienced combined vision and hearing loss. In a European study of older people aged 50 years and over 5.9\% reported DSL (Viljanen et al., 2013). The significance and potential impact of DSL in older age groups was highlighted by Brennan and Bally (2007) who estimated the prevalence of DSL in those aged 70 years and over as ranging between 5 and $20 \%$. They concluded that by 2030 , based on population ageing trends, between 3.5 and 14 million older people in the US would develop DSL.

DSL is an acquired condition, which gradually deteriorates over time. DSL is poorly understood (Davila et al., 2009), underrecognized and under-diagnosed (Heine et al., 2002). This is particularly the case in its mild form since it may be undetected by the individual, or onset may initially be in one domain at a time (vision or hearing deterioration). Older adults with DSL are more likely to have health problems, reduced activities and restricted social roles as compared to those with no sensory loss or unisensory loss (Crews and Campbell, 2004). The consequences of DSL (even mild DSL) are significant and include psychosocial difficulties, withdrawal from communication-based situations (Heine et al., 2002), avoidance of social interactions and diminished quality of life (Dalton et al., 2003; Brennan et al., 2006).

The World Health Organization estimates that $20 \%$ of adults aged 60 years and over suffer from a mental or neurological disorder (World Health Organisation, 2013a). The most common mental health disorders in older adults are dementia, depression and anxiety. Approximately $7 \%$ of older people worldwide suffer from unipolar depression and about 3.8\% of older people suffer from anxiety disorders (World Health Organisation, 2013a). Depression is a serious mental health disorder that influences quality of life and although it is known to be associated with DSL, few researchers have investigated its association and impact. In a cross sectional study of adults aged over 55 years, CapellaMcDonnall (2005) estimated that of the $7.3 \%$ of the sample who experienced DSL, 35\% experienced depression suggesting a significant relationship between DSL and depression. This finding 
has been corroborated by other researchers such as Chou and Chi (2004), Chou (2008), and Harada et al. (2008).

The literature investigating DSL in older adults is limited, although numerous studies have been published investigating a wide range of other health issues in ageing populations. A contributing factor to the paucity of research on DSL in older people is that DSL is a multisensory disorder, and requires the collaboration of various diverse professional disciplines to assess and manage the disorder. Public awareness of DSL is also not widespread and in turn, rehabilitation options have not been well-explored in many countries. Exceptions to this are discussed in papers emerging from the US, Canada and Australia (Heine and Browning, 2002; Saunders and Echt, 2007, 2012; Wittich et al., 2012).

To date, no systematic reviews of the relationship between mental health and DSL have been conducted. A review of the research is thus timely so that the association between DSL and mental health issues in older adults can be identified and used to inform programs and policies to promote healthy ageing in older people. A systematic review of the literature can also provide health professionals with evidence-based knowledge about the impacts of DSL in older adults and assist them in designing programs that will contribute to improving health outcomes in their older clients.

The aim of this systematic review was to critically review and summarize the evidence from studies that examined DSL and mental health issues (in particular depression, anxiety, and wellbeing) in older adults and evaluate the quality of the evidence by comparing it to six areas of the STrengthening the Reporting of Observational studies in Epidemiology (STROBE) statement (University of Bern, 2009).

\section{METHODS \\ EVIDENCE ACQUISITION \\ Literature search}

In accordance with Preferred Reporting Items for Systematic Reviews (PRISMA) recommendations (Moher et al., 2009), from March-November 2012, the databases Medline, PsycINFO, Cochrane Library, Sociological abstracts and Scopus were searched for relevant studies (articles from 1990 onwards were searched). The search was updated in January 2013. Groups of thesaurus terms as well as free terms were used to search the databases. Terms for older adults (thesaurus terms OR elderly, seniors, aging or ageing) were used in AND-combination with each of the terms vision and hearing loss, vision and hearing impairment, DSL or dual sensory impairment. Thereafter studies were searched for the terms mental health, anxiety, and depression. Additional articles were identified by manually searching known articles in the area of DSL.

\section{Inclusion criteria and selection process}

In order to be included in the review, studies were required to meet the following criteria:

(1) Focused on older adults (included adults at least 60 years and above);
(2) Reported vision and hearing loss as a combined construct; and

(3) Focused on an aspect of mental health.

Only full-text, peer-reviewed articles written in English were considered for inclusion. Titles, keywords, and abstracts of articles identified through the search process were reviewed to identify eligible papers. The first author initially checked eligible papers to exclude articles that were out of the scope of this study. Subsequently, the first and second author independently reviewed all potentially relevant references for eligibility. Disagreements between these reviewers were discussed with a third person (a research assistant) and a consensus decision was made.

\section{DATA EXTRACTION AND QUALITY ASSESSMENT}

The first author extracted data on the study population. On the basis of the design and methodology of individual studies, quality descriptors and ratings were derived using the STROBE statement appraisal system. The STROBE statement includes six areas for evaluation that need to be considered when appraising research, namely, the title and abstract, introduction (background/rationale and objectives), methods (study design, setting, participants, variables, data sources/measurement, bias, study size, quantitative variables and statistical methods), results (participants, descriptive data, outcome data, main results, and other analyses), discussion (key results, limitations, interpretation, generalizability) and other information (such as funding). Each study was appraised according to these areas.

\section{EVIDENCE SYNTHESIS Study selection}

The literature searches yielded 5522 unique potentially relevant articles (see Figure 1). A total of 5092 articles were classified as out of scope due to: duplication of articles, the sample did not include people aged 60 years and over (for example, included younger people with deaf-blindness due to Ushers Syndrome), the study did not focus on sensory loss, the study did not include original data and the article was not based on an empirical study. After excluding the records out of scope, the full text of each of the remaining 886 records was checked. In total, 793 (89\%) of these 886 articles did not meet the inclusion criteria. The most common reasons for primary exclusion were that the article focused only on vision impairment $(n=489,55.2 \%)$ or hearing impairment alone $(n=261,29.4 \%)$. The 93 relevant articles that remained were subject to secondary screening. The most common reasons for secondary exclusion were the focus in the results section on separate sensory losses $(n=29,31.2 \%)$ rather than a combined dual sensory construct or a focus on DSL but not on mental health impacts $(n=26,27.9 \%)$ Finally, eight studies were included in this review. Figure 1 shows the study selection process and Table 1 summarizes the studies included in the review.

\section{RESULTS AND DISCUSSION}

The eight reviewed studies were analyzed according to the STROBE statement.

The following results were obtained: 


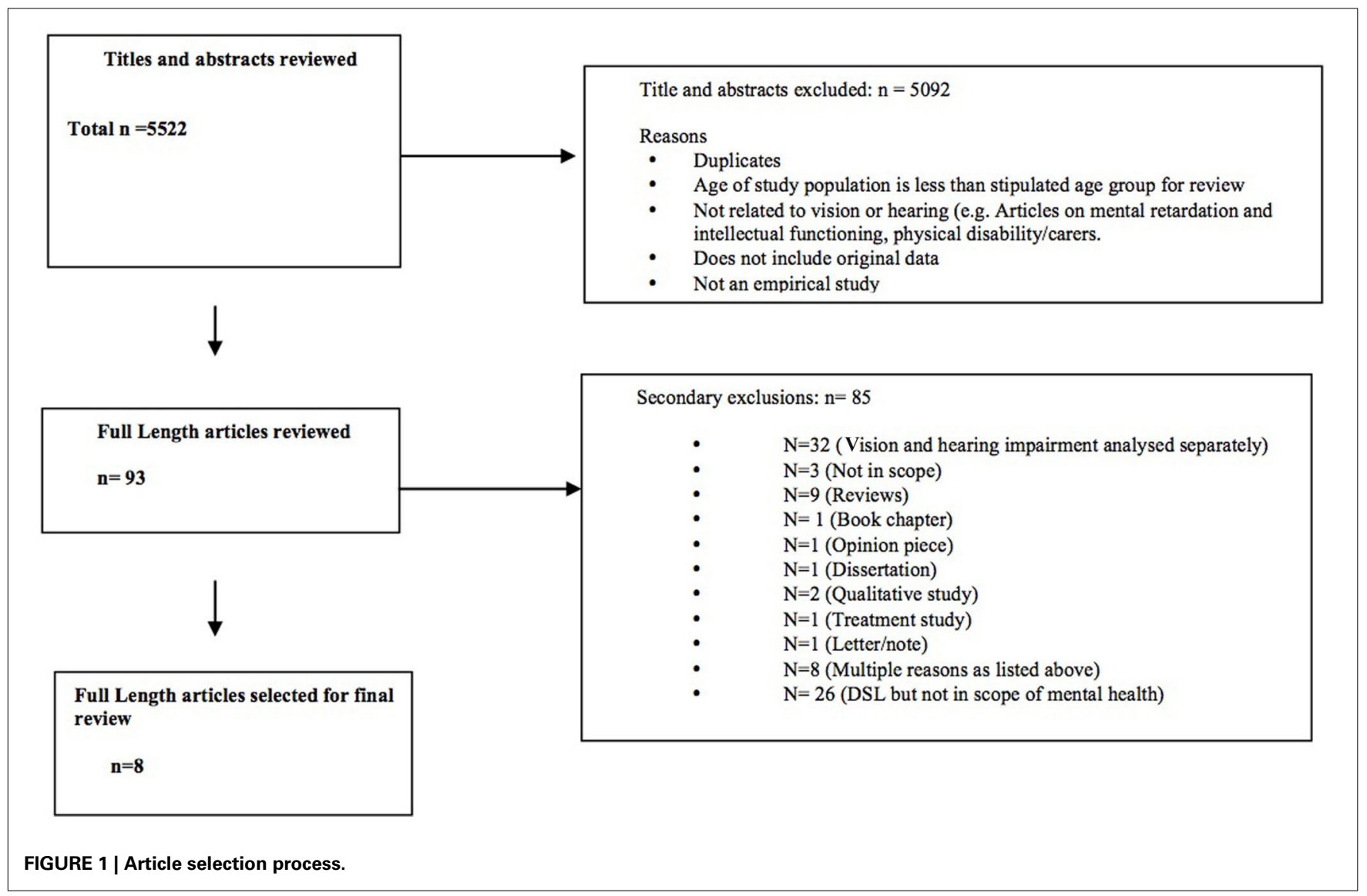

\section{RATIONALE AND OBJECTIVES}

The majority of the studies reviewed $(n=7)$ aimed to investigate the association between sensory loss (including vision loss, hearing loss and DSL) and depression or depressive symptoms. Harada et al. (2008) evaluated the impact of additional variables such as self rated health and functional ability. One study focussed specifically on the impact of hearing impairment, vision impairment and DSL on quality of life as measured by the SF36 (Chia et al., 2006). Overall, all eight studies adequately described the aims of the study.

\section{METHODS}

\section{Study population}

Overall, there were five studies that only included older participants (defined as 60 years and over for the purposes of this review) and reported the results in a way that enabled specific conclusions to be drawn about DSL in older groups (Lupsakko et al., 2002; Chou and Chi, 2004; Chia et al., 2006; Chou, 2008; Harada et al., 2008). The remaining three studies (Capella-McDonnall, 2005; McDonnall, 2009a,b) included both young and old participants. These studies examined the effects of DSL in the full sample and did not differentiate between younger and older age groups in the analyses.

\section{Sample size}

The sample size in the selected studies ranged from 203 participants to samples in which data was primarily derived from large population-based studies that included up to 9832 participants (although not all were in the older adult age range or had DSL since they were primarily prevalence studies of, for example, age related conditions). For example, in the Capella-McDonnall (2005) study, although there were 9832 participants in the unadjusted analysis and subsequently 6089 participants in the adjusted analyses, only $7.3 \%$ had DSL. Overall the sample sizes of the studies were adequate for the types of analyses performed in the respective studies.

\section{Research design}

A variety of methods were used to investigate DSL, however cross-sectional studies were the most common $(n=6)$. There were two longitudinal studies (Chou, 2008; McDonnall, 2009a). Chou (2008) used 2-year prospective data from the English Longitudinal Study of Ageing and McDonnall (2009a) used data from 9 waves of the US Health and Retirement Study. Chia et al. (2006) examined 5-year vision and hearing impairment follow up data from a baseline study of vision impairment. Two studies were designed specifically to investigate the impacts of DSL in older people (Chou and Chi, 2004; Harada et al., 2008) while the remaining studies were based on larger general studies of older people including the US Health and Retirement Study, the English Longitudinal Study on Aging, the US National Health Interview Survey, the Finnish Kuopio 75+ study and the Australian Blue Mountains Eye Study. 


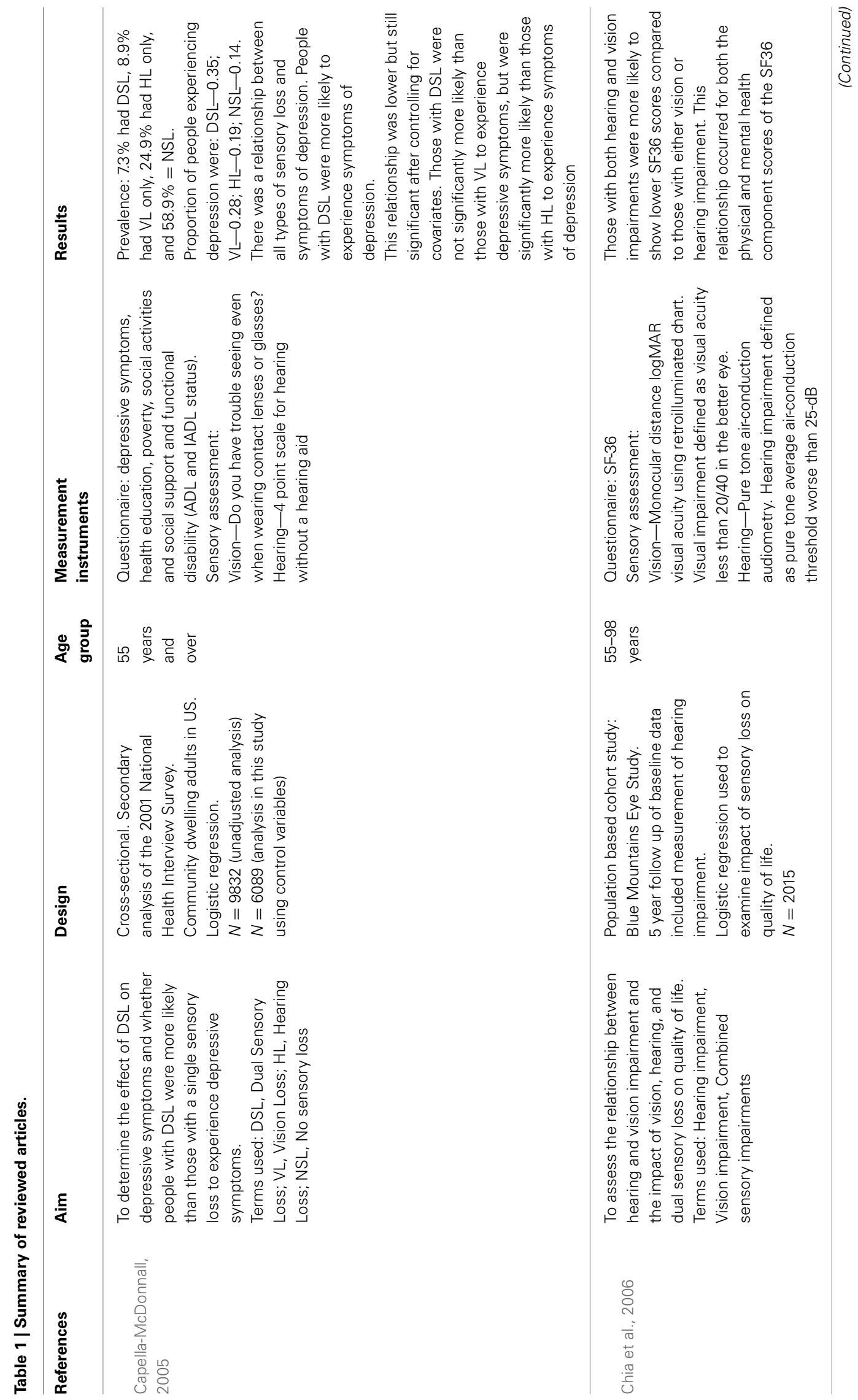




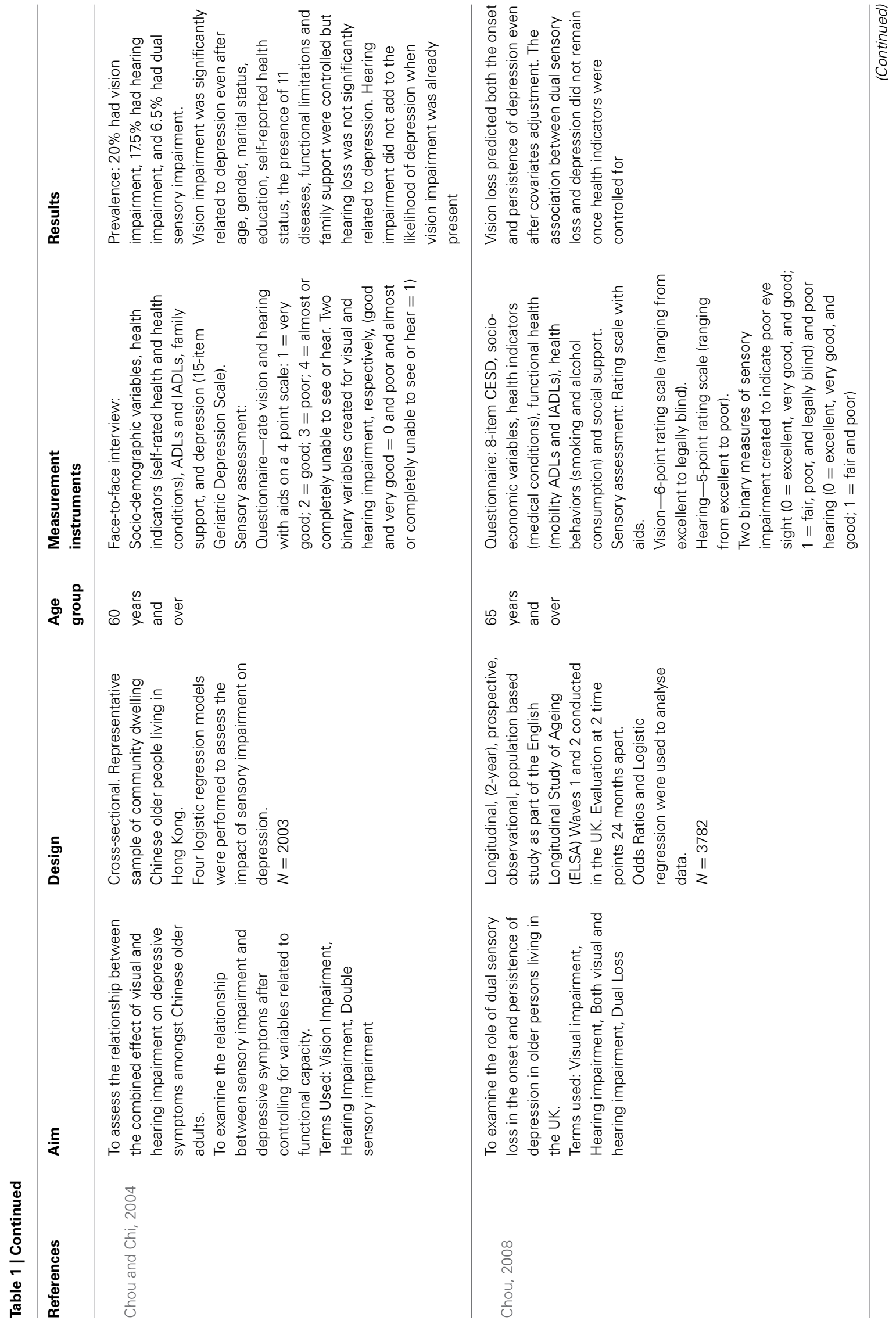




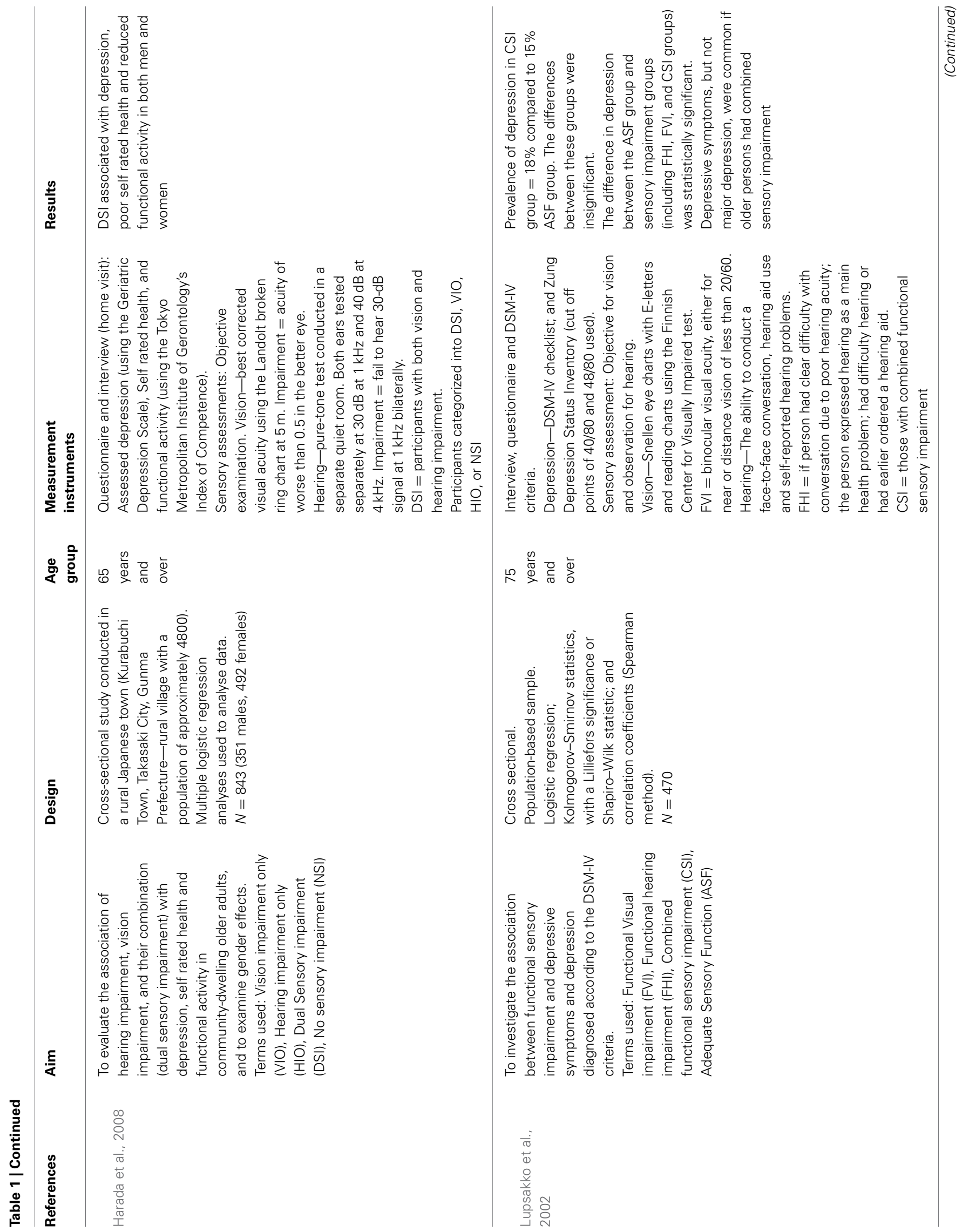



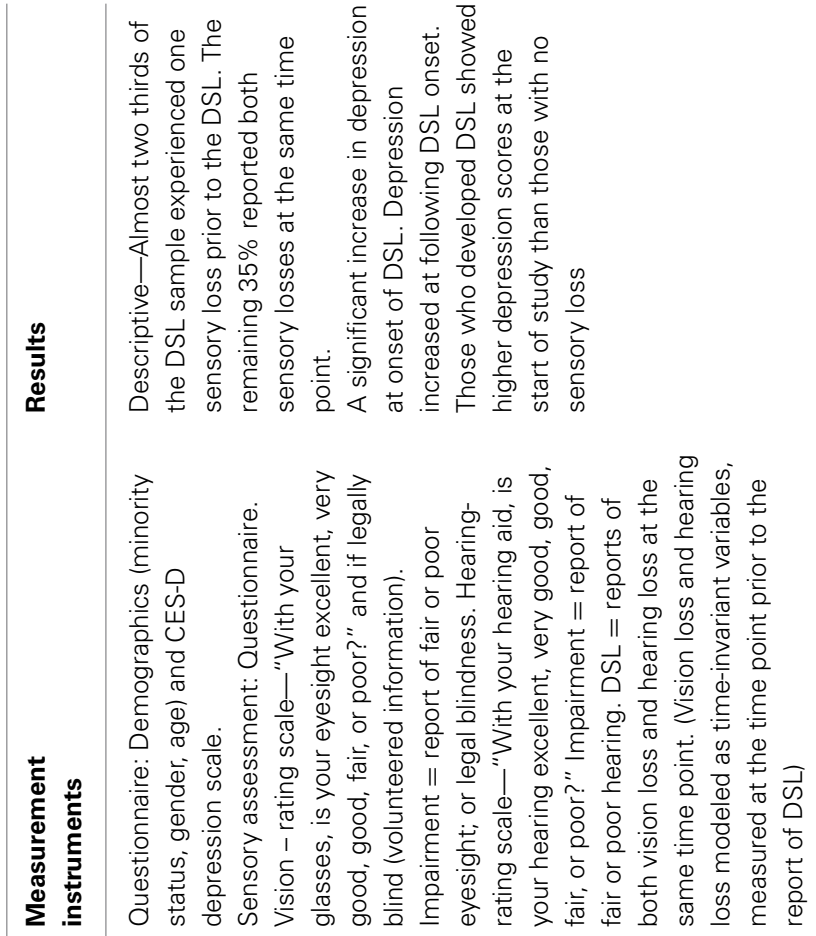

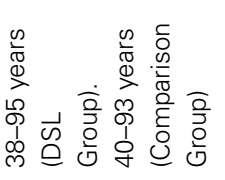

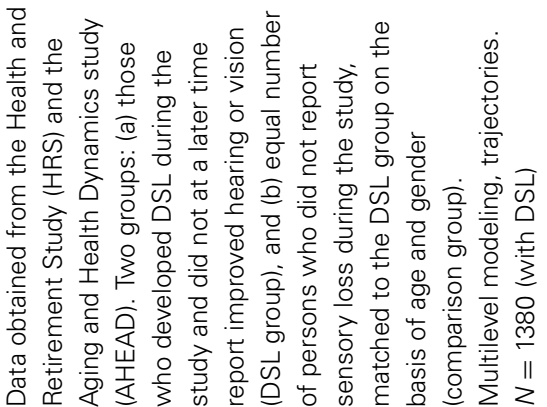

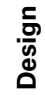

量言

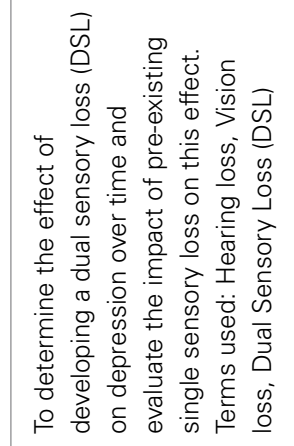

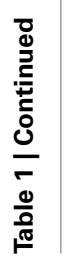

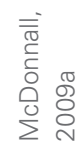

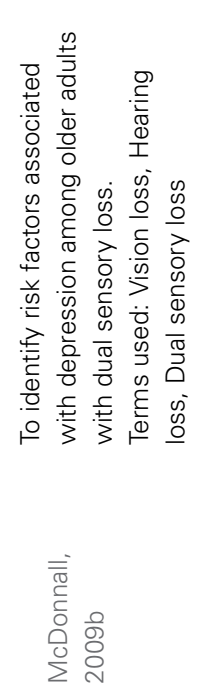
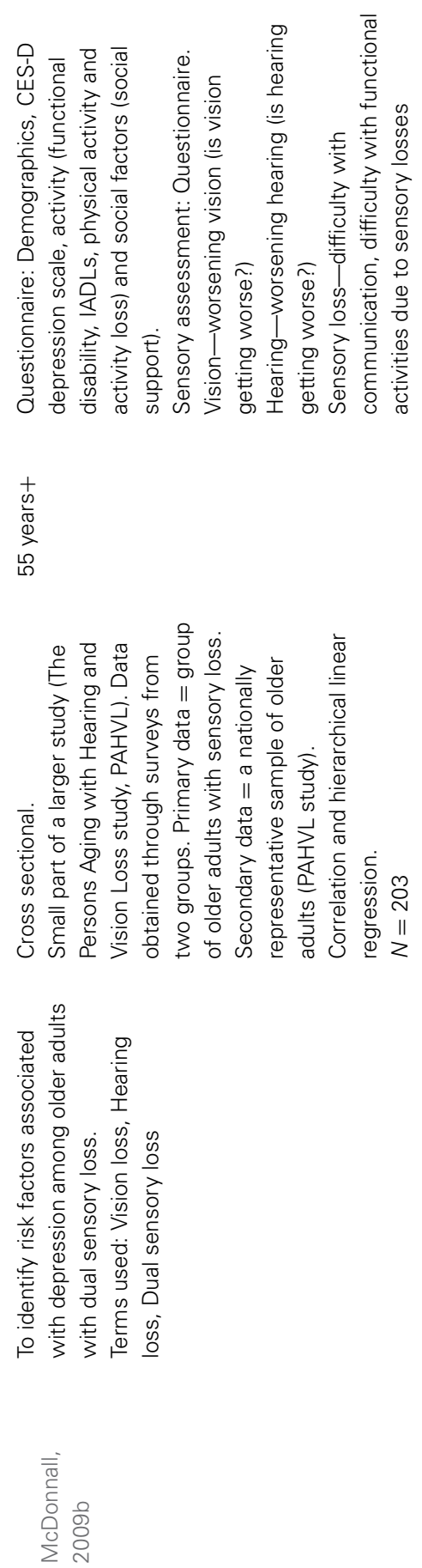

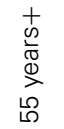

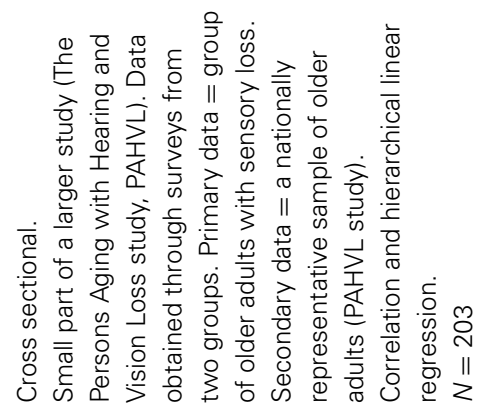




\section{Recruitment strategy}

All studies achieved well on this criterion. Participants in reviewed studies were mostly community dwelling, accessed via door-to-door interview, telephone or at senior centers. Only one study was conducted in a rural population (Harada et al., 2008). Countries were well-represented with three studies conducted in the US (Capella-McDonnall, 2005; McDonnall, 2009a,b) and one study in each of the following countries: Japan (Harada et al., 2008), Finland (Lupsakko et al., 2002), Hong Kong (Chou and Chi, 1999), UK (Chou, 2008), and Australia (Chia et al., 2006). All studies included men and women however no studies specifically reported results for any minority ethnic groups within the study country.

\section{Data collection-measurement of sensory loss and mental health variables}

There were varied methods used to obtain information relating to vision and hearing loss. Primarily, self-reported difficulty, or completion of a rating scale, were used to evaluate the presence of sensory loss. There were six studies that evaluated self-reported vision and self-reported hearing difficulty (Lupsakko et al., 2002; Chou and Chi, 2004; Capella-McDonnall, 2005; Chou, 2008; McDonnall, 2009a,b) with one study (Lupsakko et al., 2002) using observations of the ability to conduct a face-to-face conversation, hearing aid use and self-reported hearing problems. Objective measures (primarily the Snellen visual acuity test) were used to evaluate visual acuity in two studies (Lupsakko et al., 2002; Chia et al., 2006). Pure-tone audiometry was used in two studies to measure hearing acuity (Chia et al., 2006; Harada et al., 2008).

Measurement of mental health also varied across the studies. Seven of the studies either measured depression or the occurrence of depressive symptoms. One study (Capella-McDonnall, 2005) used a questionnaire to evaluate depressive symptoms, whilst six studies used a standardized scale to evaluate depression. Most commonly, the CES-D was used (Chou, 2008; McDonnall, 2009a,b) with the Geriatric Depression Scale (Chou and Chi, 2004; Harada et al., 2008) also being used. One study used two measurements to evaluate mental health. Lupsakko et al. (2002) utilized the DSM-IV checklist and Zung Depression Status Inventory. Chia et al. (2006) measured quality of life using the SF36 but not depression. In all studies, data collection methods were well-described.

\section{Data analysis}

Multiple methods were used to analyse the data including descriptive and inferential statistics such as multivariate analysis. Most commonly, regression analysis was used to examine the relationships between sensory impairment and mental health (Lupsakko et al., 2002; Chou and Chi, 2004; Capella-McDonnall, 2005; Chia et al., 2006; Chou, 2008; Harada et al., 2008; McDonnall, 2009a,b). Multilevel modeling was used to show the effects of developing DSL over time in one study (McDonnall, 2009a).

\section{RESULTS AND DISCUSSION OF REVIEWED STUDIES: FINDINGS}

Overall all studies provided a clear statement of findings relevant to this review. In relation to DSL, Capella-McDonnall (2005) estimated that out of a total sample of 6089 participants, $7.3 \%$ had
DSL of whom 35\% had depression. Although the prevalence of DSL was consistent with the findings of Chou and Chi (2004) who found that $6.5 \%$ of their sample (community dwelling, Chinese participants aged 60 years and over) had DSL, these later authors found that vision impairment was significantly related to depression whilst hearing loss was not. Furthermore, Harada et al. (2008) found that DSL was also associated with poor health and reduced functional activity. DSL was associated with lower scores on both the mental health and physical health component scores of the SF36 compared to those with hearing or vision impairment (Chia et al., 2006). In relation to the development of depression and depressive symptoms, McDonnall (2009a) found a significant increase in depression at the first report of DSL, with depression increasing at a significantly faster rate following DSL. However, participants adjusted to the impairments over time. Lupsakko et al. (2002) concluded that depressive symptoms, but not major depression, are common in older people with DSL and McDonnall (2009b) found that almost $40 \%$ of the variation in depression was due to DSL.

\section{CONCLUSION}

The aims of this systematic review were to critically review and summarize the evidence from studies that examined the mental health of older adults with DSL and evaluate the quality of the evidence by comparing it to the STROBE statement. Most of the studies reviewed provided clear aims for their study, collected their data appropriately, described the method and data analysis well, provided valid findings and were applicable to this review and therefore contributed significantly to the evidence base concerning DSL and mental health in older adults. However, some studies that included younger adults did not differentiate the effects of DSL between different age groups.

Evaluation of the studies revealed that the selected studies were primarily cross-sectional in nature and based on large population-based studies not specifically designed to investigate the association between DSL and mental health in older people. Studies primarily collected sensory impairment data via selfreport and less commonly by standardized vision and hearing acuity measurement. Depression, measured by a standardized depression scale, was the most common mental health variable investigated. Well-being was less frequently explored and there were no studies that investigated anxiety in participants with DSL.

In order to better understand the impacts of DSL on mental health in older people future research should address a number of methodological issues. It is important that future studies use both objective and subjective measures of DSL. A related issue is the definition of DSL. Studies vary in impairment cut off points for both hearing and visual acuity and while there is no obvious gold standard we would welcome further discussion and consensus on this issue. With increasing longevity and the heterogeneity of the ageing process future studies should include samples across the full older age. Very little is known for example about the impact of DSL in people in their eighties and nineties where other significant co-morbidities (including cognitive impairment) may interact with sensory loss to impact mental health. Comparative studies focussing on the relative impact of no sensory loss, single and DSL are needed to explore whether sensory loss has additive 
effects or whether older people adapt to multiple losses. While longitudinal studies are expensive, such approaches are important for understanding more fully the causal relationships between DSL and mental health outcomes in old age. Finally while a focus on the impact of DSL on depression is important other mental health problems such as anxiety should be included in future studies. Anxiety is under-researched in the older population even though there is now evidence that anxiety is more prevalent than depression in community samples of older adults (Bryant et al., 2013).

\section{AUTHOR CONTRIBUTIONS}

Both authors conceptualized and designed the review. Author A drafted the paper and Author B revised the paper. Both authors have final approval of the published article and both authors agree to be accountable for all aspects of the work in ensuring that questions related to the accuracy or integrity of any part of the work are appropriately investigated and resolved.

\section{ACKNOWLEDGMENTS}

The authors acknowledge the contribution of Dr. Marissa Dickins in the technical preparation of the paper.

\section{REFERENCES}

Brennan, M., and Bally, S. J. (2007). Psychosocial adaptations to dual sensory loss in middle and late adulthood. Trends Amplif. 11, 281-300. doi: $10.1177 / 1084713807308210$

Brennan, M., Su, Y. P., and Horowitz, A. (2006). Longitudinal associations between dual sensory impairment and everyday competence among older adults. J. Rehabil. Res. Dev. 43, 777-792. doi: 10.1682/JRRD.2005.06.0109

Bryant, C., Mohlman, J., Gum, A., Stanley, M., Beekman, A. T. F., Wetherell, J. L., et al. (2013). Anxiety disorders in older adults: looking to DSM5 and beyond. Am. J. Geriatr. Psychiatry 21, 872-876. doi: 10.1016/j.jagp.2013.01.011

Caban, A. J., Lee, D. J., Gomez-Marin, O., Lam, B. L., and Zheng, D. D. (2005). Prevalence of concurrent hearing and visual impairment in US adults: the national health interview survey, 1997-2002. Am. J. Public Health 95, 1940-1942. doi: 10.2105/AJPH.2004.056671

Capella-McDonnall, M. E. (2005). The effects of single and dual sensory loss on symptoms of depression in the elderly. Int. J. Geriatr. Psychiatry 20, 855-861. doi: $10.1002 /$ gps. 1368

Chia, E. M., Mitchell, P., Rochtchina, E., Foran, S., Golding, M., and Wang, J. J. (2006). Association between vision and hearing impairments and their combined effects on quality of life. Arch. Ophthalmol. 124, 1465-1470. doi: 10.1001/archopht.124.10.1465

Chou, K. L. (2008). Combined effect of vision and hearing impairment on depression in older adults: evidence from the english longitudinal study of ageing J. Affect. Disord. 106, 191-196. doi: 10.1016/j.jad.2007.05.028

Chou, K.-L., and Chi, I. (1999). Determinants of life satisfaction in hong kong chinese elderly: a longitudinal study. Aging Mental Health 3, 328-335. doi: $10.1080 / 13607869956109$

Chou, K. L., and Chi, I. (2004). Combined effect of vision and hearing impairment on depression in elderly Chinese. Int. J. Geriatr. Psychiatry 19, 825-832. doi: $10.1002 /$ gps. 1174

Crews, J. E., and Campbell, V. A. (2004). Vision impairment and hearing loss among community-dwelling older Americans: implications for health and functioning. Am. J. Public Health 94, 823-829. doi: 10.2105/AJPH.94.5.823

Dalton, D. S., Cruickshanks, K. J., Klein, B. E., Klein, R., Wiley, T. L., and Nondahl, D. M. (2003). The impact of hearing loss on quality of life in older adults. Gerontologist 43, 661-668. doi: 10.1093/geront/43.5.661

Dammeyer, J. (2013). Characteristics of a Danish population of adults with acquired deafblindness receiving rehabilitation services. Br. J. Vis. Impairment 31, 189-197. doi: 10.1177/0264619613490518
Davila, E. P., Caban-Martinez, A. J., Muennig, P., Lee, D. J., Fleming, L. E., Ferraro, K. F., et al. (2009). Sensory impairment among older US workers. Am. J. Public Health 99, 1378-1385. doi: 10.2105/AJPH.2008.141630

Harada, S., Nishiwaki, Y., Michikawa, T., Kikuchi, Y., Iwasawa, S., Nakano, M., et al. (2008). Gender difference in the relationships between vision and hearing impairments and negative well-being. Prev. Med. 47, 433-437. doi: 10.1016/j.ypmed.2008.06.011

Heine, C., and Browning, C. (2002). Communication and psychosocial consequences of sensory loss in older adults: overview and rehabilitation directions. Disabil. Rehabil. 24, 763-773. doi: 10.1080/09638280210129162

Heine, C., Erber, N., Osborn, R., and Browning, C. (2002). Communication perceptions of older adults with sensory loss and their communication partners: implications for intervention. Disabil. Rehabil. 24, 356-363. doi: $10.1080 / 09638280110096250$

Lupsakko, T., Mantyjarvi, M., Kautiainen, H., and Sulkava, R. (2002). Combined hearing and visual impairment and depression in a population aged 75 years and older. Int. J. Geriatr. Psychiatry 17, 808-813. doi: 10.1002/gps.689

McDonnall, M. C. (2009a). The effects of developing a dual sensory loss on depression in older adults: a longitudinal study. J. Aging Health 21, 1179-1199. doi: $10.1177 / 0898264309350077$

McDonnall, M. C. (2009b). Risk factors for depression among older adults with dual sensory loss. Aging Mental Health 13, 569-576. doi: 10.1080/13607860902774410

Moher, D., Liberati, A., Tetzlaff, J., and Altman, D. for The PRISMA Group. (2009). Preferred reporting items for systematic reviews and meta-analyses: the PRISMA statement. BMJ 339, 332-336. doi: 10.1136/bmj.b2535

Saunders, G. H., and Echt, K. V. (2007). An overview of dual sensory impairment in older adults: perspectives for rehabilitation. Trends Amplif. 11, 243-258. doi: 10.1177/1084713807308365

Saunders, G. H., and Echt, K. V. (2012). Blast exposure and dual sensory impairment: an evidence review and integrated rehabilitation approach. J. Rehabil. Res. Dev. 49, 1043-1058. doi: 10.1682/JRRD.2010.08.0157

Schneider, J., Gopinath, B., McMahon, C., Teber, E., Leeder, S., Wang, J. J., et al. (2012). Prevalence and 5-year incidence of dual sensory impairment in an older Australian population. Ann. Epidemiol. 22, 295-230. doi: 10.1016/j.annepidem.2012.02.004

Smith, S. L., Bennett, L. W., and Wilson, R. H. (2008). Prevalence and characteristics of dual sensory impairment (hearing and vision) in a veteran population. J. Rehabil. Res. Dev. 45, 597-609. doi: 10.1682/JRRD.2007.02.0023

University of Bern. 2014. (2009). STROBE Statement [cited January 15 2014]. Available online at: http://www.strobe-statement.org/

Viljanen, A., Törmäkangas, T., Vestergaard, S., and Andersen-Ranberg, K. (2013). Dual sensory loss and social participation in older Europeans. Eur. J. Ageing. 11, 155-167. doi: 10.1007/s10433-013-0291-7

Wittich, W., Watanabe, D. H., and Gagne, J. P. (2012). Sensory and demographic characteristics of deafblindness rehabilitation clients in Montreal, Canada. Ophthalmic Physiol. Opt. 32, 242-251. doi: 10.1111/j.1475-1313.2012.00897.x

World Health Organisation. 2014. (2013a). Mental Health and Older Adults [cited January 14 2014]. Available online at: http://www.who.int/mediacentre/ factsheets/fs381/en/

World Health Organisation. (2013b). What is Mental Health? Available online at: http://www.who.int/features/qa/62/en/index.html

Conflict of Interest Statement: The authors declare that the research was conducted in the absence of any commercial or financial relationships that could be construed as a potential conflict of interest.

Received: 03 February 2014; accepted: 17 April 2014; published online: 14 May 2014. Citation: Heine C and Browning CJ (2014) Mental health and dual sensory loss in older adults: a systematic review. Front. Aging Neurosci. 6:83. doi: 10.3389/fnagi. 2014.00083

This article was submitted to the journal Frontiers in Aging Neuroscience.

Copyright (c) 2014 Heine and Browning. This is an open-access article distributed under the terms of the Creative Commons Attribution License (CC BY). The use, distribution or reproduction in other forums is permitted, provided the original author(s) or licensor are credited and that the original publication in this journal is cited, in accordance with accepted academic practice. No use, distribution or reproduction is permitted which does not comply with these terms. 